\title{
Quality Improvement During Gastroenterology Fellowship: The Experience of Improving Inpatient Post-endoscopy Communication
}

\author{
Amy G. Ogurick ${ }^{1}$ Samantha J. Magier ${ }^{2} \cdot$ Michelle L. Hughes $^{1}$
}

Accepted: 2 February 2022 / Published online: 3 March 2022

(c) The Author(s), under exclusive licence to Springer Science+Business Media, LLC, part of Springer Nature 2022

\section{Summary Box}

- Although GI fellows are required to participate in QI projects during training, they often lack the appropriate resources, guidance, or inspiration to complete a meaningful project

- Identification of frequently frustrating clinical situations or inefficiencies in daily workflow may provide the impetus for an impactful QI project that can be feasible and fellow-driven if standard QI methodology is followed

- An example is given of the implementation of a standardized post-inpatient procedure brief operative note template that significantly improved the timing and quality of post-procedural documentation while improving communication between the GI consultant and the primary team

\section{Problem Identification}

Quality improvement (QI) initiatives are an iterative process aimed at, amongst other things, improving patient outcomes through timely, effective care within a healthcare system [1]. In 2014, the Accreditation Council for Graduate Medical Education (ACGME) implemented a formal requirement for all trainees, which includes gastroenterology (GI) fellows, to participate in QI initiatives [2]. Despite this mandate, there continues to be significant inter-program variability in the extent and nature of trainee participation due to lack of formal training and mentorship in quality improvement

Michelle L. Hughes

michelle.hughes@yale.edu

1 Section of Digestive Diseases, Yale University School of Medicine, New Haven, CT, USA

2 Department of Internal Medicine, Yale University School of Medicine, New Haven, CT, USA coupled with the stresses inherent in balancing clinical work, research efforts, and procedural training for the busy fellow [3].

Gastroenterology offers many opportunities for trainees to improve quality and patient safety through simple, relevant projects that can improve their day-to-day experiences [4, 5]. In order to engage GI trainees in these initiatives, it is important that selected projects are meaningful to patients, as well as to the trainee, and can be completed within a reasonable timeframe. Trainees should select projects that address one of the six dimensions for healthcare quality that aim to provide safe, effective, timely, efficient, equitable, and patient-centered care [6]. Any of these six dimensions can be applied as a lens to focus on improving daily challenges and annoyances such as repetitive overnight pages, workflow inefficiencies or redundancies, or barriers to patient care. Importantly, by focusing on issues that affect the day-to-day provision of care, trainees are more likely to stay engaged and develop skills to continue sustainable improvement initiatives in their future practices following graduation.

A key part of daily life for GI fellows serving on an inpatient consult service is post-endoscopy communication. Timely and accurate documentation of endoscopic findings and post-procedure recommendations in the electronic medical record (EMR) is essential in an inpatient service, especially where handoffs are frequent. At our institution, procedure notes do not populate into the EMR until they are signed by the attending physician, which may not happen immediately, and are uploaded to an area different than used for the inpatient notes written by other services due to limitations in the endoscopy software interface. These barriers created challenges for primary teams who were frequently left without timely written guidance on post-procedure management, resulting in provider dissatisfaction, confusion, delays in care, and multiple after-hours pages to on-call fellows who were often not directly involved in the patient's care. To circumvent this, some GI fellows would enter a 
brief post-procedure note directly into the EMR, although the use and quality of these notes were inconsistent.

Our initiative had three major aims. The first aim was to improve the quality of post-procedure documentation with respect to consistent recommendations for diet, proton-pump inhibitor (PPI) use, the resumption of anticoagulants (AC) or antiplatelet (AP) medications, and the institution of antibiotic therapy. The second aim was to improve the timeliness of post-endoscopy documentation by our inpatient GI consultant team. Lastly, we aimed to improve perceived covering provider satisfaction of GI consultant post-procedure communication.

\section{Searching for Solutions}

There is evidence both within the surgical and GI literature that standardized notes using tools such as templates can help improve quality of post-procedure communication [7-9]. In order to address issues surrounding periprocedural communication, GI faculty, fellows, and internal medicine providers, were identified as key stakeholders and asked to provide input into the development of a new note template utilizing kaizen, a "lean" methodology principle that emphasizes joint efforts of employees in order to continuously improve the workplace. Specifically, the practice of kaizen "blitz" was performed, which crowdsources stakeholder ideas to address problems and propose incremental and ultimately sustainable solutions through rapid iterative improvement cycles [10]. Utilizing this feedback, a standardized brief-operative note template was developed intended to be entered directly into the EMR following inpatient endoscopy in the same location as are other inpatient notes. The note was intended to be completed in one to two minutes by utilizing a drop-down structure to minimize additional burden to busy GI providers and was paired with education regarding template use. This note served as the first intervention of our improvement cycle (Fig. 1). An improvement cycle, otherwise known as a plan-do-study-act (PDSA) cycle, is the framework of a QI project to evaluate an intervention in real-world settings.

A retrospective before-after study was performed to determine completeness of documentation following endoscopy for adult inpatients. Due to the importance of capturing variability in real-world settings when planning QI initiatives, pre-intervention or background data was collected from four non-consecutive weeks in order to detect variations that may occur between groups of fellows as they rotate on inpatient consults every six to twelve weeks. For the first rapid improvement cycle, post-intervention data were collected each Friday (since Friday was identified as the point of the week where handoff is most important) for ten consecutive weeks after the template was introduced. Charts of patients who underwent any inpatient endoscopy were reviewed. Data from these charts was collected, including the procedure time, time of posting of the brief procedure note (if written), time of posting of the final procedure note, and whether essential recommendations were addressed. The indication for PPI or AC was based on a review of the patient's listed medications at the time of the hospital encounter; information from the initial GI consult note was determined by the authors who collected data (AO and SM). Initial background data were collected on these three metrics; however, based on the principles key to QI including rapid-cycle improvement and kaizen blitz feedback, the decision was made to subsequently include AP and antibiotic recommendations within the template. Therefore, these were included in the implementation of the template, though preand post-intervention data on these specific metrics were not collected.

Statistical analysis was performed using two-tailed t-tests and chi-squared tests to compare documentation between the two time periods. A survey of primary team perceptions of the GI service post-procedure communication was administered pre-and post-intervention to internal medicine inpatient providers including trainees, hospitalists, and advanced practice providers. Aims of the intervention included completion of post-procedure documentation, timeliness of documentation, and primary provider perception.

Process, balancing, and outcome measures, the metrics used in QI initiatives, were also identified [6]. A process measure evaluates how the changes made to fix steps within a process are working or being utilized to affect desired outcomes. In this project, the process measures were: 1)time to post-procedure documentation, 2) use of the new note template, and 3) completion of all template components. Balancing measures assess the potential unintended negative consequences of an intervention, such as the additional workload of completing an extra note in this case. An additional balancing measure identified was the use of generic recommendations from the drop-down menu rather than patient-specific recommendations. For example, selecting "resume systemic anticoagulation" is confusing if selected for a patient not previously receiving AC. These balancing measures were not explicitly measured in this initial first cycle of the project. Outcome measures evaluate how interventions impact the ultimate project aim. In this project, though patient-related outcomes are the measure of interest, they have not yet been collected since additional improvement cycles are needed to be completed before new data categories can be collected.

\section{Assessing the Impact}

In total, 157 pre-intervention and 92 post-intervention inpatient endoscopic procedures were evaluated. There was a significant improvement in the use of brief operative notes (pre: 


\begin{tabular}{|c|c|c|}
\hline Brier ost-Procieaure o & Noie & \\
\hline Procedure(s) Performed: & $\begin{array}{l}\text { EGD } \\
\text { Colonoscopy } \\
\text { Flexible Sigmoidoscopy } \\
\text { EGD/Push enteroscopy } \\
\text { EGD with PEG placement }\end{array}$ & $\begin{array}{l}\text { Video capsule endoscopy } \\
\text { EUS } \\
\text { ERCP } \\
\text { EGD with stent placement } \\
\end{array}$ \\
\hline
\end{tabular}

Summary of endoscopic findings:

\#**

Recommendations:

Diet Advance diet to full prior diet

Advance diet as tolerated, starting with clear liquids and advance to full prior diet

Clear liquid diet until further instructed by GI team

Maintain NPO until further instructed by GI team

***

PPI use

Transition to PPI from IV to PO BID

Transition $\mathrm{PPI}$ from $\mathrm{IV}$ to $\mathrm{PO}$ once daily

Continue IV PPI BID $x 72$ hours post-procedure then can transition to PO PPI BID

Continue current PPI dosing and route

Discontinue PPI

$* * \pi+$

Svstemic anticoagulation

Resume systemic anticoagulation at prior dosing if clinically indicated

Resume anticoagulation if clinically indicated, but recommend use of high-risk, no bolus heparin drip and monitoring for recurrence of bleeding Recommend holding systemic anticoagulation until further instructed by $\mathrm{Gl}$ team

xᄎะ

N/A

Antiplatelet use

Resume or continue aspirin Hold any other antiplatelet agents (if previously taking

Resume or continue all antiplatelet agents

N/A

Antibiotic use

Continue current antibiotics for ${ }^{* i \mathrm{k}}$ days

No ongoing antibiotics required from $\mathrm{Gl}$ standpoint

*a*x

N/A

Other Recommendations

Avoid NSAIDS

Continue octreotide drip for total of *** hours post-procedure and then start non-selective beta-blocker as tolerated

Discontinue octreotide drip

Follow-up pathology

Labs: $\mathbf{x x \pi}$

Imaging: ${ }^{* * *}$

Consults: ${ }^{x \times x}$

Repeat endoscopy: ${ }^{* * *}$

Contact Gl fellow on call if $* * *$

***

NAA

\begin{tabular}{|l|}
\hline Patient updated with findings \\
\hline Patient's family updated with findings \\
Primary team updated with findings \\
\hline$\pi \approx *$
\end{tabular}

Final procedure note will be available in Epic under Chart Review -> "Procedures" tab once completed by attending with finalized findings and recommendations.

Fig. 1 Template created for brief post-endoscopy note. Yellow boxes represent pull-down where only one option can be selected. Blue boxes represent pull-down where multiple options can be selected. $* * *$ designates area where provider can free-text

49.7\% vs post: $90.2 \% p<0.001)$ as well as documentation of relevant clinical recommendations (pre: $53.8 \%$ vs post: $89.2 \%, p<0.001$ ) (Table 1). There was significant improvement in documentation of post-procedure dietary recommendations (pre: $65.4 \%$ vs post: $96.3 \%, p<0.001$ ) and anticoagulation management recommendations (pre: $50.0 \%$ vs post: $89.3 \%, p=<0.01)$. Recommendations regarding PPI management did not significantly improve post-intervention, although pre-intervention utilization was already high (pre: $84.6 \%$ vs post: $92.5 \% \mathrm{p}=0.39$ ).

There was improvement of documentation prior to shift change or within two hours of procedure completion (pre: $65.6 \%$ vs post: $79.3 \%, p=0.03$ ), as well as improvement in primary team provider's perception of post-procedure 
Table 1 Post-procedure documentation. $\mathrm{PPI}=$ proton pump inhibitor, $\mathrm{AC}=$ anticoagulation

\begin{tabular}{lccc}
\hline Metric & Pre-intervention & Post-intervention & $p$-value \\
\hline Brief operative note written & & & \\
Combined services & $78 / 157(49.7 \%)$ & $83 / 92(90.2 \%)$ & $p<0.001$ \\
General GI & $62 / 98(63.3 \%)$ & $61 / 62(98.4 \%)$ & $p<0.001$ \\
Advanced & $16 / 59(27.1 \%)$ & $22 / 30(73.3 \%)$ & $p<0.001$ \\
Procedure documentation prior to shift change or within 2 h of procedure completion & \\
Combined services & $103 / 157(65.6 \%)$ & $73 / 92(79.3 \%)$ & $p=0.03$ \\
General GI & $78 / 20(79.60 \%)$ & $54 / 62(87.1 \%)$ & $p=0.32$ \\
Advanced & $25 / 34(42.4 \%)$ & $19 / 30(63.3 \%)$ & $p=0.10$ \\
Brief operative note addressed relevant clinical \\
recommendations (combined services) & $42 / 78(53.2 \%)$ & $74 / 83(89.2 \%)$ & $p<0.001$ \\
Diet & & & \\
PPI & $51 / 78(65.4 \%)$ & $79 / 82(96.3 \%)$ & $p<0.001$ \\
AC & $33 / 39(84.6 \%)$ & $49 / 53(92.5 \%)$ & $p=0.39$ \\
\hline
\end{tabular}

communication following template education and implementation (Fig. 2). Nevertheless, formal statistical analysis was not performed on the subjective survey data.

\section{Reflecting and Refining}

Implementation of a standardized post-procedure brief operative note template by GI providers following inpatient endoscopy significantly improved the timing and quality of post-procedure documentation, improving primary team perception of communication. This was the first cycle of an ongoing QI initiative aimed at improving interdisciplinary communication following inpatient endoscopy. A strength of this project was that it benefited key stakeholders while mitigating additional burdens on time and effort, a likely reason for its rapid uptake and engagement. The use of the template, which conveyed timely and standardized recommendations and findings, was perceived as beneficial to primary teams while the use of this focused note template immediately following a procedure was determined to be a feasible intervention for improvement.
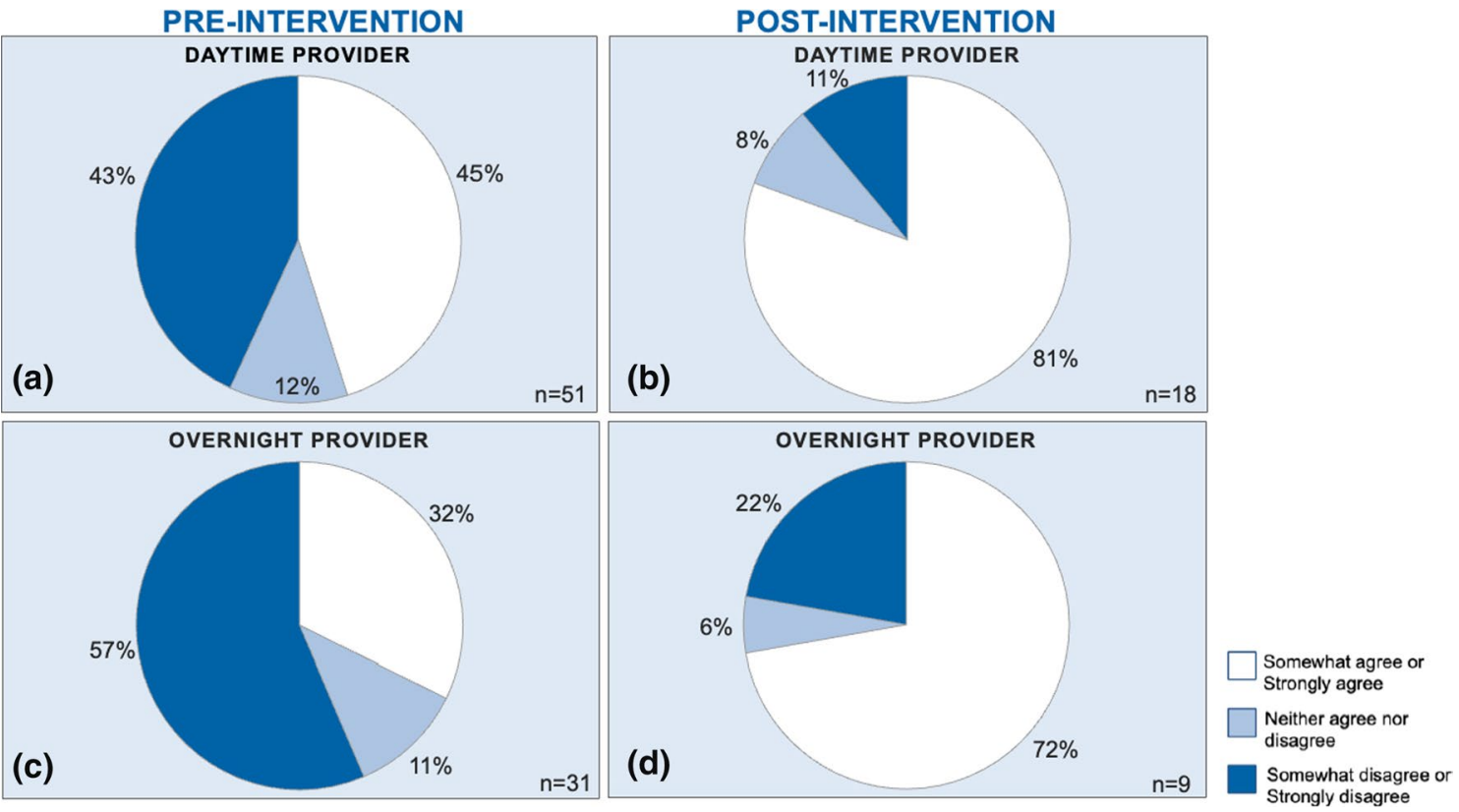

Fig. 2 Primary team providers were asked to comment on the degree to which they agreed with the statement "While in the role of daytime primary team provider $(\mathrm{a} / \mathrm{b})$ or overnight covering provider (c/d), I feel that GI procedure information is clearly communicated and accessible including post-procedure recommendations such as diet and medication recommendations." These results are averaged between the responses for the general GI service and the advanced endoscopy service 
After a quality improvement cycle, the decision is made if the intervention is ready to be fully adopted, if it needs to be adapted and further polished prior to widespread adoption, or if it should be abandoned as ineffective or infeasible. We determined that though a standardized post-procedure template was an effective intervention, it needed to be further modified before widespread adoption in order to ensure that its recommendations are accurate. The first cycle revealed the frequent use of generic recommendations surrounding $\mathrm{AC}$ rather than useful patient-specific guidance, felt to be in part due to the difficulty of accessing medication lists during certain phases of hospital care. Future cycles will improve the template in order to leverage the EMR and automate this information, reducing reliance on memory and further streamlining the process. Following additional cycles, outcome measures will be analyzed to ascertain how these process measures translate to direct patient care outcomes, such as length of stay considerations and re-initiation of appropriate post-endoscopy medications and diet.

This project, practical for GI fellows to devise and implement, can meaningfully positively impact patient care. QI is a valuable approach for trainees to initiate and study an intervention within the timeframe of their training. Many institutions have IRB exemptions or expedited processes for QI work that helps ensure completion during training. Trainees should seek mentors familiar with QI and attempt to take on realistically scaled projects while considering the six dimensions of health care quality when brainstorming QI ideas [6]. Interventions can be small obviating the requirement for large-scale implementation or funding. Changes are meant to be studied in the real-world environment not only for real-time feedback and decisions but also in order to ensure sustainable processes and outcomes. Trainees can use free resources such as the Institute for Healthcare Improvement (IHI) Open School or Agency for Healthcare Quality and Research (AHRQ) to garner skills and become involved in quality improvement [6]. Using this framework, trainees should find that they can complete an impactful project for patients and the healthcare system alike.

\section{References}

1. Easler JJ. Quality improvement for the gastroenterology fellow. Gastrointest Endosc. 2013;77:281-283. https://doi.org/10.1016/j. gie.2012.10.027.

2. Accreditation Council for Graduate Medical Education. ACGME Common Program Requirements. July 1, 2017. Accessed December 27, 2021. http://www.acgme.org/Portals/0/PFAssets/Progr amRequirements/CPRs_2017-07-01.pdf

3. Koh NJ, Wagner R, Newton RC et al. Detailed findings from the CLER national report of findings 2018. J Grad Med Educ. 2018;10:49-68. https://doi.org/10.4300/1949-8349.10.4s.49.

4. Germansky KA, Leffler DA. Development of quality measures for monitoring and improving care in gastroenterology. Best Pract Res Clin Gastroenterol. 2011;25:387-395. https://doi.org/10. 1016/j.bpg.2011.05.00.

5. Siddique SM, Ketwaroo G, Newberry C, Mathews S, Khungar V, Mehta SJ. How to incorporate quality improvement and patient safety projects in your training. Gastroenterology. 2018;154:1564-1568. https://doi.org/10.1053/j.gastro.2018.03. 044.

6. Institute for Healthcare Improvement. Open School. Updated July 7, 2020. Accessed December 27, 2021. http://www.ihi.org.educa tion/ihiopenschool/Pages/default.aspx

7. Harris N, Telford J, Yonge J, et al. Improvement of Endoscopic Reports with Implementation of a Dictation Template. J Can Assoc Gastroenterol. 2019;4(1):21-26. Published 2019 Dec 19. https://doi.org/10.1093/jcag/gwz033

8. Cromwell PM, Flood M, O Connell E, et al. POST-OP: A Strategy to Improve Clinical Documentation in the Early Postoperative Period. J Surg Educ. 2018;75(4):957-963. https://doi.org/10. 1016/j.jsurg.2018.01.005

9. Shaikh FA, Alvi AR, Shahzad N, Gala T, Murtaza G. Template operative note, a better documentation. J Pak Med Assoc. 2019;69:409-411.

10. Mazzocato P, Stenfors-Hayes T, von Thiele Schwarz U, Hasson H, Nyström ME. Kaizen practices in healthcare: a qualitative analysis of hospital employee' suggestions for improvement. BMJ Open. 2016;6(7):e012256. Published 2016 Jul 29. Doi:https://doi.org/ 10.1136/bmjopen-2016-012256

Publisher's Note Springer Nature remains neutral with regard to jurisdictional claims in published maps and institutional affiliations. 